\title{
Diet-derived nutrient patterns and components of metabolic syndrome: a cross-sectional community- based study
}

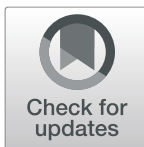

Mahdi Vajdi ${ }^{1}$, Mahdieh Abbasalizad Farhangi ${ }^{2^{*}}$ and Leila Nikniaz ${ }^{3}$

\begin{abstract}
Background: Metabolic syndrome (MetS) is one of the main public health problems worldwide. Although some relations between dietary intakes and MetS have been found, few studies have focused on association between dietary nutrients interactions and the risk of the MetS and its components. The aim of the present study was to examine the association between nutrient patterns and MetS and its components among Iranian adult population.

Methods: A total of 588 subjects (aged 18-64 years, 271 males and 317 females) enrolled in the cross sectional study. Dietary consumption was evaluated using an 80-item food frequency questionnaire (FFQ). Biochemical assessments including fasting blood sugar (FBS), aspartate aminotransferase (AST), alanine amino transferase (ALT) and serum lipids were performed by enzymatic methods. Nutrient patterns were obtained by factor analysis procedure using principal component method. Multinational logistic regression analysis was used to evaluate the association between nutrient patterns and MetS and its components.

Results: Three nutrient patterns explaining $53.66 \%$ of the variance in dietary nutrients intake, were recognized in the current study. Animal-sourced nutrient pattern was significantly associated with the higher odds of MetS and high triglyceride (TG) levels. Plant-sourced nutrient pattern (high intake of fiber, carbohydrate, vitamins $B_{6}, B_{3}, C_{,} B_{1}$, E, D, magnesium, potassium, and linoleic acid) was significantly associated with lower risk of MetS and lower blood pressure $(p<0.05)$. Third nutrient pattern (mixed-source) was significantly related to higher risk of MetS, high waist circumference (WC) and high systolic blood pressure (SBP).

Conclusion: This present study confirms the important effect of nutrients and their patterns on MetS risk. Our results suggest that adherence to the nutrient pattern rich in fiber, carbohydrate, vitamins $D, B_{6}, B_{3}, C, B_{1}, E$, magnesium, potassium, linoleic acid, and docosahexaenoic acid (DHA) is associated with a lower risk of MetS, while animal- and mixed-sourced nutrient patterns are positively associated with greater odds of MetS; However, further longitudinal and interventional studies are required to make a clear conclusion.
\end{abstract}

Keywords: Obesity, Nutrient pattern, Factor analysis, Metabolic syndrome, Iranian population

\footnotetext{
* Correspondence: abbasalizadfarhangim@gmail.com

${ }^{2}$ Drug Applied Research Center, Tabriz University of Medical Sciences, Tabriz, Iran

Full list of author information is available at the end of the article
}

(c) The Author(s). 2020 Open Access This article is licensed under a Creative Commons Attribution 4.0 International License, which permits use, sharing, adaptation, distribution and reproduction in any medium or format, as long as you give appropriate credit to the original author(s) and the source, provide a link to the Creative Commons licence, and indicate if changes were made. The images or other third party material in this article are included in the article's Creative Commons licence, unless indicated otherwise in a credit line to the material. If material is not included in the article's Creative Commons licence and your intended use is not permitted by statutory regulation or exceeds the permitted use, you will need to obtain permission directly from the copyright holder. To view a copy of this licence, visit http://creativecommons.org/licenses/by/4.0/ The Creative Commons Public Domain Dedication waiver (http://creativecommons.org/publicdomain/zero/1.0/) applies to the data made available in this article, unless otherwise stated in a credit line to the data. 


\section{Background}

The metabolic syndrome (MetS) is characterized by a clustering of cardiovascular risk factors, including central obesity, elevated blood pressure, dyslipidemia (high triglyceride and low high-density lipoprotein cholesterol (HDLC)) and fasting hyperglycemia [1]. The prevalence of MetS has been increasing worldwide and it has become a main public health problem in numerous countries.

The MetS prevalence varies from 11.6 to $62.5 \%$ around the worldwide [2] and according to a recent systematic review and meta-analysis the prevalence of MetS in Iran was 35 and $24 \%$ in women and men, respectively [3].

The etiology of MetS is complex and involves the interactions between metabolic, environmental and genetic factors [4]. In addition to ethnic predisposition, physical activity, smoking, stress and body mass index (BMI), diet, as a major lifestyle factor, may be strongly associated with the development of MetS [5]. Dietary intervention is a fundamental strategy for the prevention and treatment of MetS [6]. In recent decades, several studies have evaluated the effect of dietary patterns and isolated foods on development of MetS $[7,8]$ and some results have proposed that consumption of vegetables and fruits is linked with a reduced risk of MetS [9, 10]. Fruits and vegetables are main contributors of magnesium, vitamin $\mathrm{C}$, potassium, and vitamin $\mathrm{A}$, and they may potentially decrease the risk of chronic life-threatening diseases [11].

There are several methods to examine the associations of dietary intake and food groups with MetS in epidemiologic studies $[12,13]$. It has previously been proposed that dietary patterns may provide valuable perception of dietdisease associations [14] and maybe more predictive of chronic disease risk than the consumption of single foods or nutrients [15]. Several researches on dietary patterns have shown that adherence to prudent, Dietary Approach to Stop Hypertension (DASH) diet, Mediterranean diet are associated with lower risk of MetS [16-19]. In contrast, a meta-analysis by Rodríguez-Monforte $\mathrm{M}$ et al. in 2017 revealed that unhealthy dietary patterns (i.e. Western diet) were positively associated with MetS [20].

Since individuals do not consume isolated nutrients but rather eat meals consisting of a variety of foods with multiple nutrients, the traditional method of assessing the diet and disease relationship, which focuses on highly correlated nutrients separately, maybe inappropriate for taking into account cumulative synergistic or interactive effects on the circulating levels, metabolism, bioavailability, and excretion of nutrients [14]. Thus, assessing the combined effect of nutrients through creating nutrient patterns could be an appropriate alternative method for assessing the effects of diet on diseases [21]. The nutrient pattern method is a combination of multiple nutrients and may provide more information about probable underlying mechanisms, interactions and synergic effects of nutrients
[15]. Several studies have found the inverse association between MetS and single nutrients such as vitamin D [22], calcium [23], vitamin C [24], carotene [25], and potassium [26]. There are limited studies conducted in Iran that evaluated the association between the nutrient patterns and MetS and only Khayyatzadeh et al. [27] have previously evaluated this relationship in Mashhad, Northeast Iran. They reported that a nutrient pattern which mostly characterized by dietary maltose, glucose, carbohydrate, sucrose, protein, starch, and fructose, was related to a higher risk of MetS in both sexes. Until now, there is no study in the literature investigating the relationship between nutrient patterns and MetS in northwest of Iran. So, the aim of this study was to recognize any nutrient patterns and subsequently, to examine the relationship between the nutrient patterns and components of MetS in northwest of Iran.

\section{Methods \\ Study population}

The data for this cross-sectional study were taken from major lifestyle promotion project (LPP) in East Azerbaijan, Iran, aimed to explore the prevalence of non-communicable diseases and the associated risk factors. Probability proportional to size multistage stratified cluster sampling was used as a sampling method. The exact method of sampling is described in our previous study [28]. Briefly, 150 clusters were selected and 590 participants from 150 clusters were enrolled. Incomplete questionnaires were excluded and the final sample subjected to analysis was 588 individuals. People were included in the current study if their original nationality was Iranian and aged between 18 and 64 years. Individuals with physical disability, severe chronic illness requiring bed rest, active liver injury, mental disability, and pregnant women were excluded. The Ethics Committee of Tabriz University of Medical Sciences approved the current study (Registration number: IR.TBZMED.REC.1398.077) and all individuals signed the written informed consent before participating in this crosssectional study. The work is obtained from M.S. thesis of Mahdi Vajdi.

\section{General characteristics and anthropometric measurements}

The anthropometric, demographic and dietary information were collected by expert health care professionals. The demographic information included age, education, physical activity, marital and smoking status. The sociodemographic characteristics and smoking status were collected through a direct interview using a questionnaire and physical activity level was evaluated using the Official Persian short form of international physical activity questionnaire (IPAQ-SF) [29]. Body weight was 
measured using a digital scale (Seca, Dubai, United Arab Emirates) while subjects minimally clothed without shoes and height was measured to the nearest $0.5 \mathrm{~cm}$ with a stadiometer. Body mass index (BMI) was then calculated by weight $(\mathrm{kg})$ divided by height squared $\left(\mathrm{m}^{2}\right)$. Blood pressure was measured with a standard mercury sphygmomanometer with a standard cuff twice in the same arm in sitting position. Waist circumference (WC) was measured in horizontal plane, midway between the iliac crest and the rib cage with a measuring tape in centimeter. Waist to hip ratio (WHR) was calculated by waist circumference divided by hip circumference.

\section{Dietary assessment}

Trained dietitians collected dietary information over the preceding 1 year through an 80-item quantitative food frequency questionnaire (FFQ), which was developed and validated for LPP study and reference portions were defined based on the most reported portion sizes in the 24-h recall [30]. Subjects were asked to report their frequency and the amount of intake for each food item on a daily, weekly, monthly or yearly basis. Reported frequency of each food item, were then converted to daily gram intake. In addition to the daily energy, 40 nutrients intake for each subject were computed using the US Department of Agriculture's (USDA) and Nutrients Composition of Iranian Foods. In the present study, we used protein, carbohydrate, fat, total dietary fiber, cholesterol, saturated fatty acids (SFAs), monounsaturated fatty acids (MUFAs), polyunsaturated fatty acids (PUFAs), vitamin $A$, vitamin $E$, vitamin $D$, vitamin $K$, thiamin, pantothenic acid, vitamin B12, biotin, riboflavin, niacin, pyridoxin, vitamin $\mathrm{C}$, folate, Alpha tocopherol, linolenic acid, linoleic acid, DHA, eicosapentaenoic acid (EPA), potassium, chromium, magnesium, sodium, iron, phosphorus, calcium, manganese, copper, fluoride, selenium, zinc, sugar and caffeine to identify nutrient patterns.

\section{Biochemical assessment}

About $10 \mathrm{ml}$ blood sample was taken from each participant after an overnight fast. All the samples were immediately centrifuged at $2000 \mathrm{rpm}$ for $10 \mathrm{~min}$ at room temperature. Fasting blood sugar (FBS) and Lipid profile [triglyceride (TG), High-Density Lipoprotein-Cholesterol (HDL-C) and total cholesterol (TC)] were evaluated using enzymatic colorimetric technique (Pars Azmoon commercial kit, Tehran, Iran) and Low-Density Lipoprotein-Cholesterol (LDL-C) was measured by Friedewald eq. [31]. Alanine aminotransferase (ALT) and aspartate transaminase (AST) were assessed using the ultraviolet method [32]. Chemiluminescent immunoassay technology was used for the quantitative determination of serum $25(\mathrm{OH})$ D $(25 \mathrm{OH}$ Vitamin D Total Assay, DiaSorin, Saluggio, Italy).

\section{Definition of the metabolic syndrome}

Participants were categorized as having MetS according to the internationally accepted criteria of NCEPATP III [33], if they have three or more of the following characteristics: $\mathrm{TG} \geq 150 \mathrm{mg} / \mathrm{dl}$ or current use of medicine for dyslipidemia; $W C \geq 88 \mathrm{~cm}$ in women and $\geq 102 \mathrm{~cm}$ in men; diastolic blood pressure (DBP) $\geq 85 \mathrm{mmHg}$ or systolic blood pressure (SBP) $\geq 130 \mathrm{mmHg}$ or using antihypertensive medication; HDL-C $<50 \mathrm{mg} / \mathrm{dl}$ in women and $<40 \mathrm{mg} / \mathrm{dl}$ in men or current use of medicine for dyslipidemia and FBS $\geq 100 \mathrm{mg} / \mathrm{dl}$ or on medication treatment for hyperglycemia.

\section{Statistical analyses}

All analyses were carried out using the Statistical Package for Social Sciences (SPSS, version 21, Chicago, IL, USA). The principal component method for factor analysis was employed to determine major nutrient patterns (called factors) based on the forty predefined nutrients and three explainable factors were kept. The reliability of the factor analysis was tested by Kaiser-Meyer-Olkin (KMO) test and orthogonal Varimax rotation was carried out to clarify the interpretability and decrease the correlation between the factors. The number of factors was determined by considering scree plot, eigenvalues $>$ 1.00 and the interpretability of the factors. Factor loadings of the nutrients in each nutrient pattern were computed. Positive loading in a factor shows a direct relationship with the factor, while a negative loading shows that the nutrient is inversely relationship with the factor. We calculated the factor score for each participant and nutrient pattern by summing up intakes of nutrients weighted by their factor loadings. For each nutrient pattern, participants were grouped into four categories according to quartiles of factor scores. Continuous variables are presented as the means \pm standard deviations, and categorical variables are expressed as the number (\%). Categorical and continuous variables were compared across quartiles of nutrient pattern scores using analysis of $\mathrm{x}^{2}$ and analysis of variance (ANOVA) tests, respectively. To find the association between nutrient patterns and odds of MetS and its components, multinational logistic regression so was used in different models. Model 1 was adjusted for sex, age, smoking, physical activity, education levels and energy intake. The first quartile was used as a reference for calculating the odds ratios (OR).

\section{Result}

The mean age of the participants was $42.26 \pm 11.97$ years and the mean BMI was $27.37 \pm 4.81 \mathrm{~kg} / \mathrm{m}^{2}$. About $46.1 \%$ of the population was male. The prevalence of high DBP, SBP, TG, WC, FBS and low HDL were 26.7, 29.4, $38.8,60.4,43.9$ and $13.6 \%$ respectively while total 
prevalence of MetS was 39.3\%. In the current study three major nutrient patterns based on 40 food components were extracted: animal-sourced pattern characterized by high intake of saturated fatty acid (SFA), fat, protein, sugar, vitamins $B_{5}, B_{12}, B_{2}, A, K$, biotin, phosphorus, cholesterol, selenium, sodium, polyunsaturated fatty acids (PUFA), calcium and zinc; plant-sourced pattern with high consumption of fiber, carbohydrate, vitamins $B_{6}, B_{3}, C, B_{1}, E, D$, magnesium, potassium, linoleic acid, and DHA and mixed-source nutrient pattern including fluoride, manganese, caffeine and folate.

The identified nutrient patterns described $53.66 \%$ of the total variance in dietary nutrient intake (Table 1). The participants' characteristics across quartiles of nutrient patterns are presented in Table 2. Across different quartiles of animal-sourced pattern, those in top quartiles had significantly higher weight, smoking habits, energy and macronutrient intake compared to the participants in the first quartile $(p<0.05)$. Within the plant-sourced pattern, those in higher quartiles had significantly higher weight, energy and macronutrient intake compared to the participants in the lower quartiles $(p<0.05)$ and those in lower quartiles had significantly higher age and higher prevalence of hypertension than compared to the participants in the higher quartiles $(p<$ 0.05). Participants in higher quartiles of mixed-source nutrient pattern had significantly higher BMI, energy and carbohydrate intake compared to the participants in the bottom quartile $(\mathrm{p}<0.05)$.

Biochemical and anthropometric characteristics of the study population across quartiles of nutrient pattern are presented in Table 3. We found that participants in the higher quartiles of the animal-sourced pattern had significantly higher DBP, TG, ferritin, and AST and the individuals in the second quartile had a higher HDL-C $(p=0.001)$ compared to first quartiles $(p<0.05)$. Within the plant-sourced pattern, participants in the higher quartile had significantly lower WC, WHR, SBP, FBS and LDL-C than those in the first quartile $(p<0.05)$. Participants in higher quartiles of mixed-source nutrient pattern had significantly higher WC and SBP B compared to the participants in the bottom quartile $(\mathrm{p}<$ 0.05).

Multivariable-adjusted Odd's ratio (OR) and confidence interval (CI) for MetS and its components across quartiles of dietary nutrient patterns are shown in Table 4. In an unadjusted model, participants in the top quartile of the animal-sourced pattern were more likely to have 2.35-fold higher odds for MetS compared with those in the first quartile (OR: $2.35,95 \% \mathrm{CI}: 1.11-5.92$ ) and those in the fourth quartile of the animal-sourced pattern tended to have 1.36-fold higher odds for higher TG compared with those in the first quartile (OR: 1.36, 95\% CI: 1.03-1.78). However, this relationship was no longer significant after controlling for sex, age, smoking status, education level and energy intake. In an unadjusted model, participants in the top quartile of the plant-sourced pattern were $50 \%$ less likely to have MetS compared with those in the first quartile (OR: 0.50, 95\% CI: 0.28-0.87). However, this relationship was no longer significant after adjustment for confounders. Also, in an unadjusted model, significant inverse associations were noted between second quartile of the plant-sourced nutrient pattern and high SBP (OR: 0.46, 95\% CI: $0.27-$ 0.78 ) and high DBP (OR: 0.45, 95\% CI: 0.27-0.77). Both in crude and adjusted regression analyses, participants in the third quartile of the mixed-source nutrient pattern were $71 \%$ more likely to have MetS (crude OR: 1.71 and adjusted OR:1.77) and tended to have 2.32-fold higher odds for higher SBP compared with those in the first quartile (OR: 2.32, 95\% CI: 1.34-4.00). In the case of WC, those in the higher quartile of mixed-source nutrient pattern had significantly higher odds for higher WC compared with those in the lowest quartile (OR: 2.51, 95\% CI: 1.55-4.06). Figure 1 presents the prevalence of components of MetS across three nutrient patterns. As shown in this Figure, having low serum HDL-C and high blood sugar had the highest and lowest prevalence among metabolic disorders of all of nutrient patterns. No difference between three nutrient patterns regarding the prevalence of metabolic disorders was reported.

\section{Discussion}

Our study is the first to examine the association between nutrient patterns and MetS and its components in north-west Iran. Considering the recent increase in MetS prevalence in Iran, there was a terrible need to search for moderating factors. Although relations between nutrients and risk of multiple chronic conditions have received increased interest in recent years but a few studies are available on the association of nutrient patterns with risk of non-communicable diseases $[8,11]$. Assessing nutrient patterns give us a snapshot of all dietary nutrients with all of their relations, which can be very important. Only one study evaluating the relationship between nutrient patterns and MetS in Iranian population. They reported that Western pattern (consisting of maltose, glucose, carbohydrate, sucrose, protein, starch, and fructose) was related to a higher risk of MetS in both sexes [27]. Our findings revealed three main nutrient patterns in East-Azerbaijan population: animal-sourced, plant sourced and mixed source nutrient pattern. We found that adherence to the "animalsource" and "mixed source" patterns were positively associated with MetS whereas plant source nutrient pattern was inversely associated the risk of MetS. Nutrients included in nutrient pattern analyses are very diverse between different studies. In this current study, we tried to 
Table 1 Factor loading matrix for the nutrients representing the three major nutrient patterns

\begin{tabular}{|c|c|c|c|}
\hline Nutrients & Animal sourced & Plant sourced & Mixed-source \\
\hline Fatty acid- saturated & 0.951 & 0.196 & - \\
\hline Fat & 0.932 & 0.253 & - \\
\hline Protein & 0.920 & 0.345 & - \\
\hline Vitamin $B_{5}$ & 0.908 & 0.361 & 0.125 \\
\hline Vitamin $B_{12}$ & 0.901 & 0.258 & - \\
\hline Phosphorus & 0.878 & 0.358 & _- \\
\hline Cholesterol & 0.828 & 0.222 & - \\
\hline Selenium & 0.812 & 0.380 & _- \\
\hline Sodium & 0.810 & 0.195 & - \\
\hline Vitamin $B_{2}$ & 0.784 & 0.453 & 0.300 \\
\hline Vitamin $\mathrm{K}$ & 0.729 & 0.292 & - \\
\hline Calcium & 0.704 & 0.171 & _- \\
\hline Zinc & 0.610 & 0.253 & - \\
\hline Sugar & 0.563 & 0.147 & 0.128 \\
\hline Vitamin A & 0.472 & 0.367 & - \\
\hline PUFA & 0.333 & 0.324 & _ \\
\hline Biotin & 0.112 & - & - \\
\hline Alpha tocopherol & - & - & - \\
\hline Chromium & - & - & - \\
\hline MUFA & - & - & - \\
\hline Copper & - & - & - \\
\hline Linolenic acid & - & - & - \\
\hline Total fiber & - & 0.891 & - \\
\hline Vitamin $B_{6}$ & 0.497 & 0.786 & _- \\
\hline Carbohydrate & 0.207 & 0.785 & 0.176 \\
\hline Vitamin $B_{3}$ & 0.538 & 0.709 & _- \\
\hline Vitamin C & - & 0.673 & - \\
\hline Magnesium & 0.453 & 0.670 & 0.497 \\
\hline Vitamin D & - & 0.633 & _- \\
\hline Vitamin $B_{1}$ & 0.153 & 0.619 & - \\
\hline Potassium & 0.327 & 0.549 & 0.443 \\
\hline Vitamin E & 0.138 & 0.468 & _ \\
\hline Linoleic acid & 0.262 & 0.293 & _- \\
\hline DHA & & 0.101 & \\
\hline Iron & - & - & - \\
\hline Fluoride & _- & _- & 0.982 \\
\hline Manganese & _- & 0.124 & 0.980 \\
\hline Caffeine & - & - & 0.976 \\
\hline Folate & 0.447 & 0.559 & 0.603 \\
\hline EPA & - & - & - \\
\hline Percentage of Variance explained & 27.60 & 16.23 & 9.82 \\
\hline
\end{tabular}

Loading values of 0.10 or greater are indicated by bold value. Abbreviations: MUFA monounsaturated fatty acid, PUFA polyunsaturated fatty acids, DHA docosahexaenoic acid, EPA eicosapentaenoic acid 


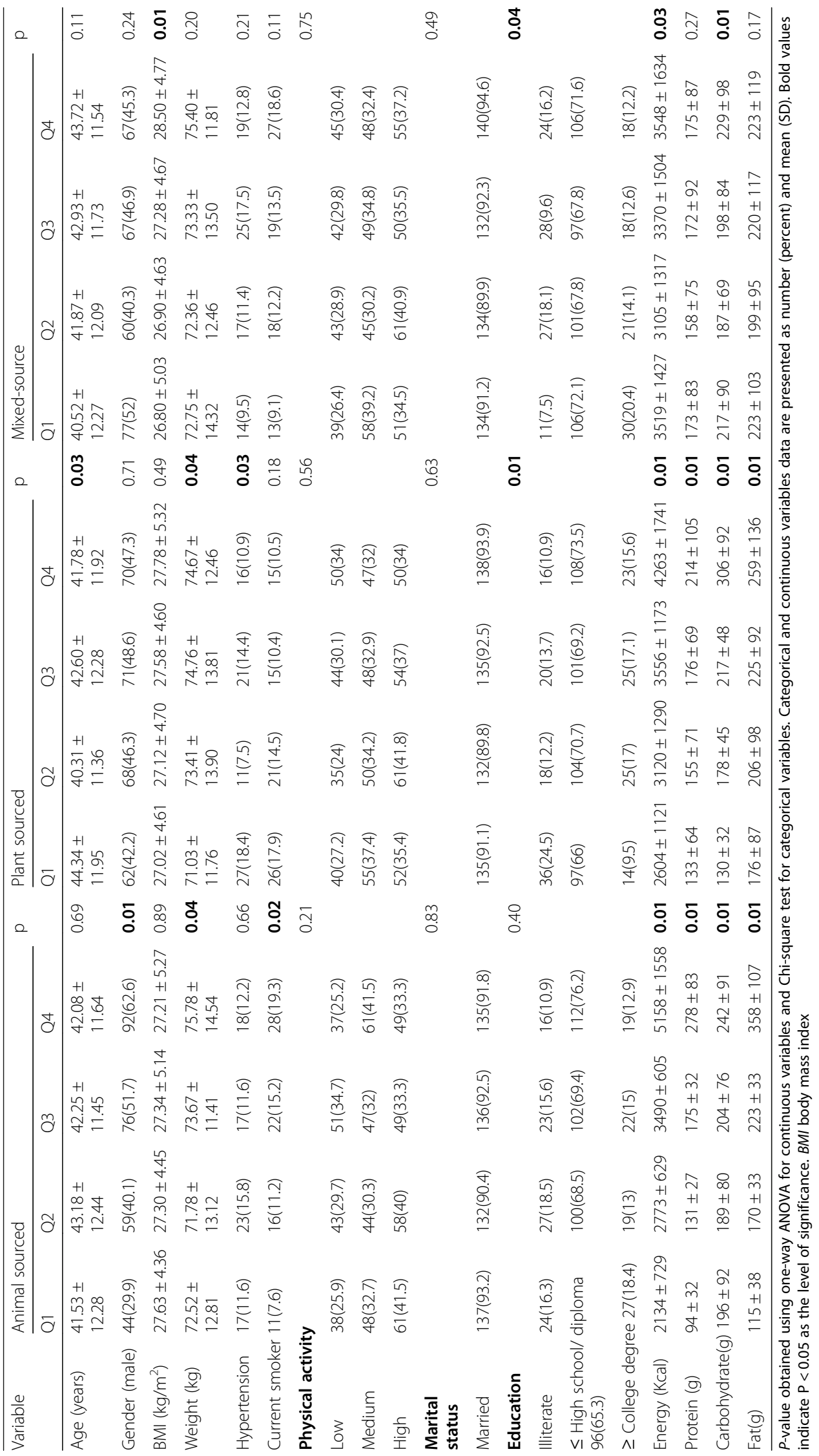




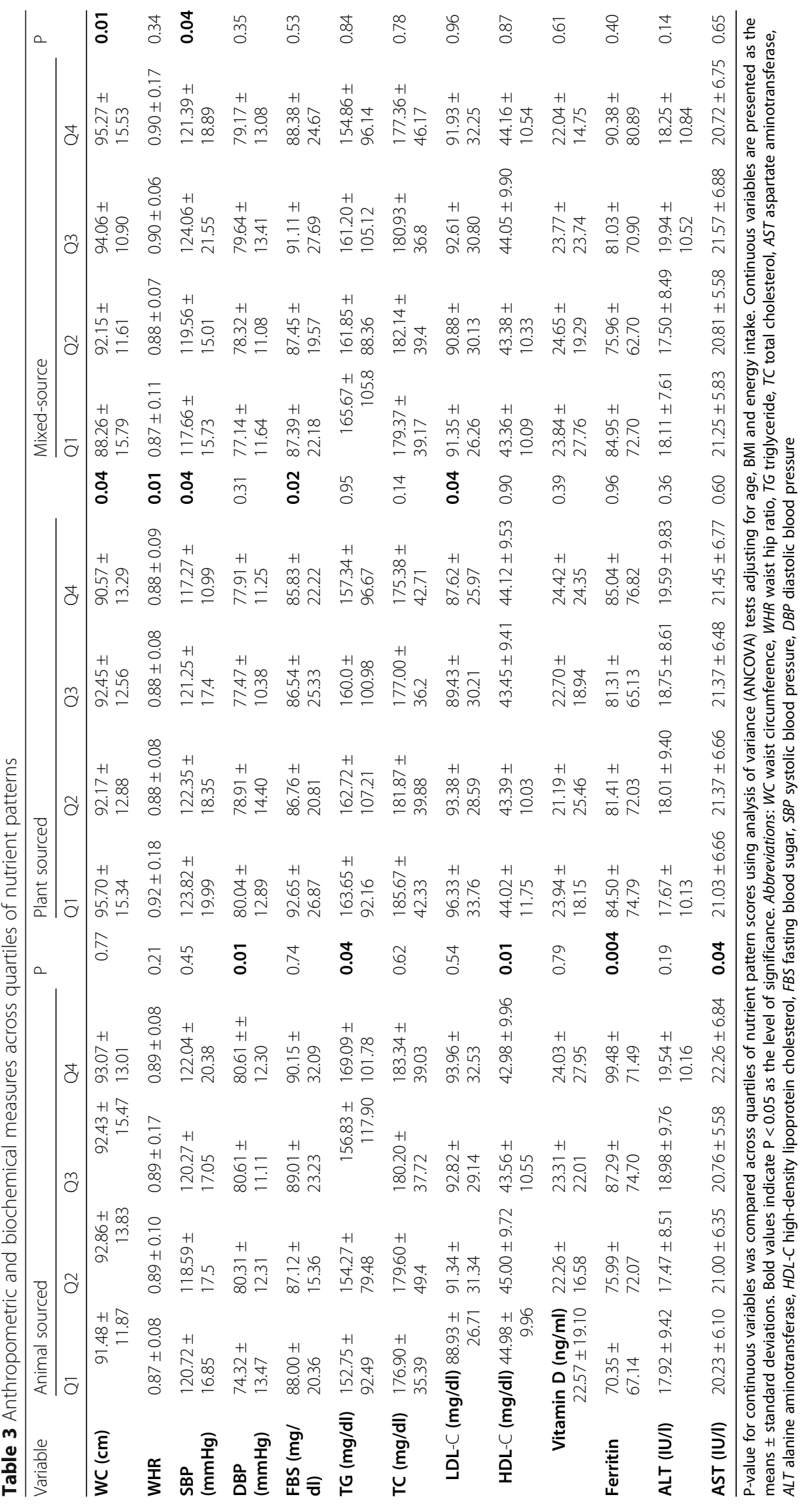




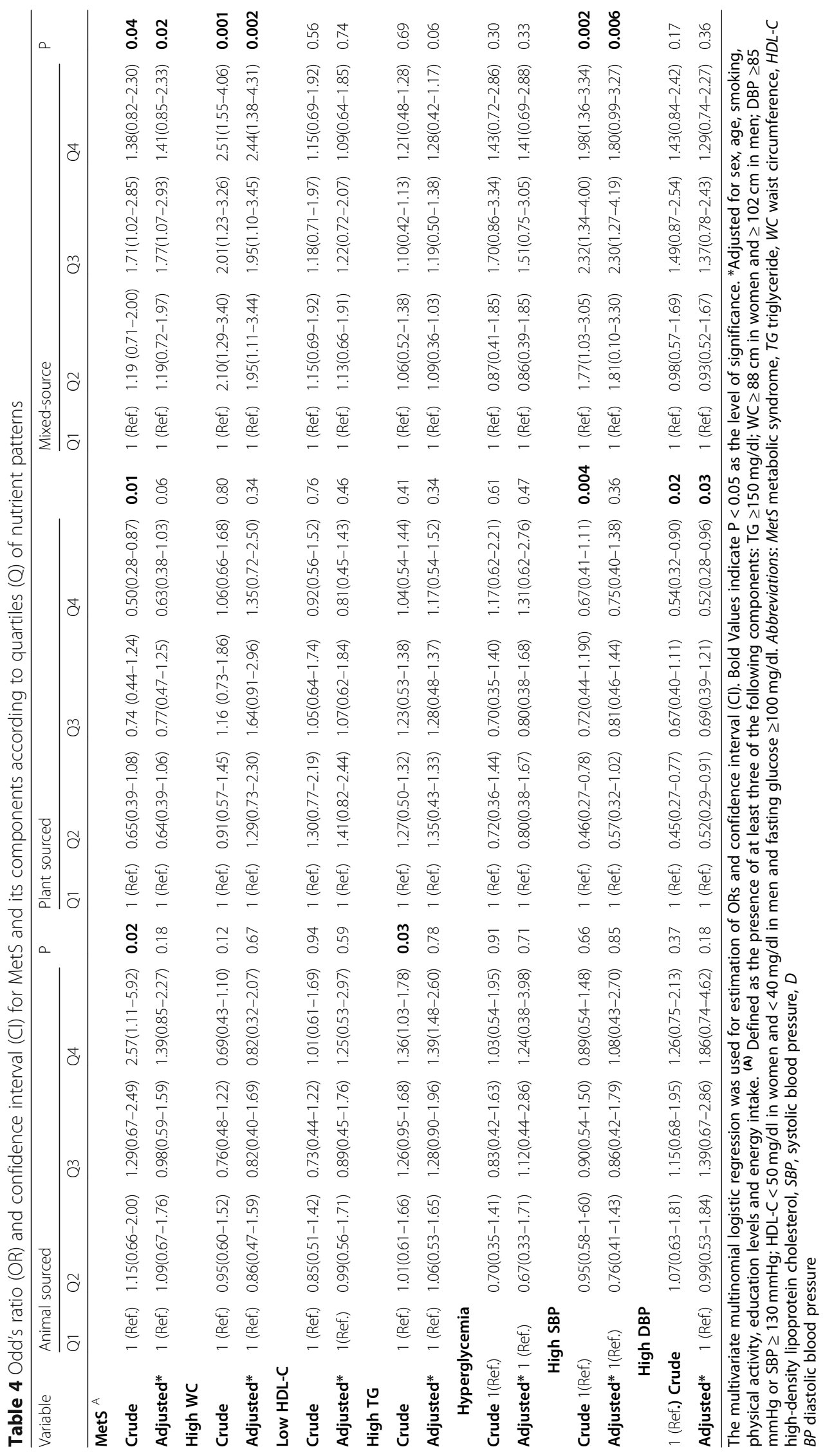




\section{A}

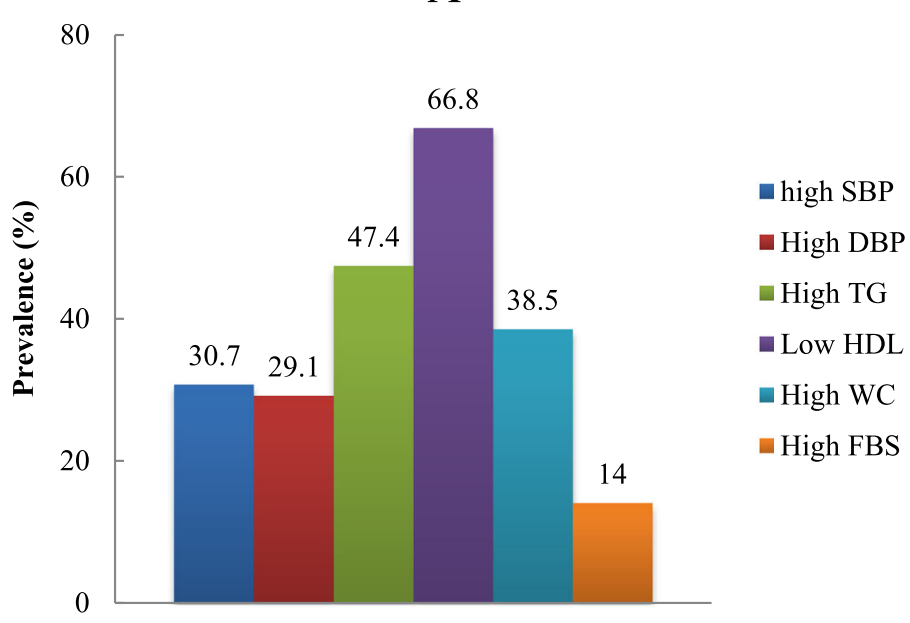

B

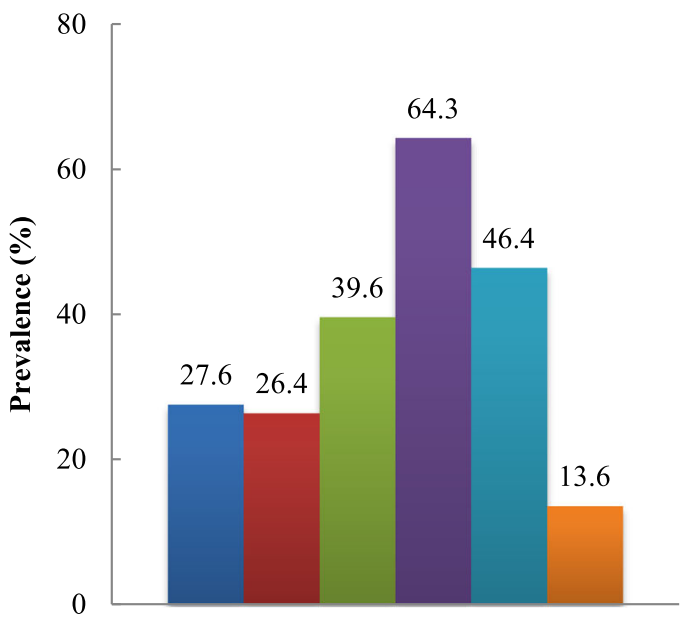

- high SBP

- High DBP

- High TG

- Low HDL

- High WC

- High FBS

$\mathrm{C}$

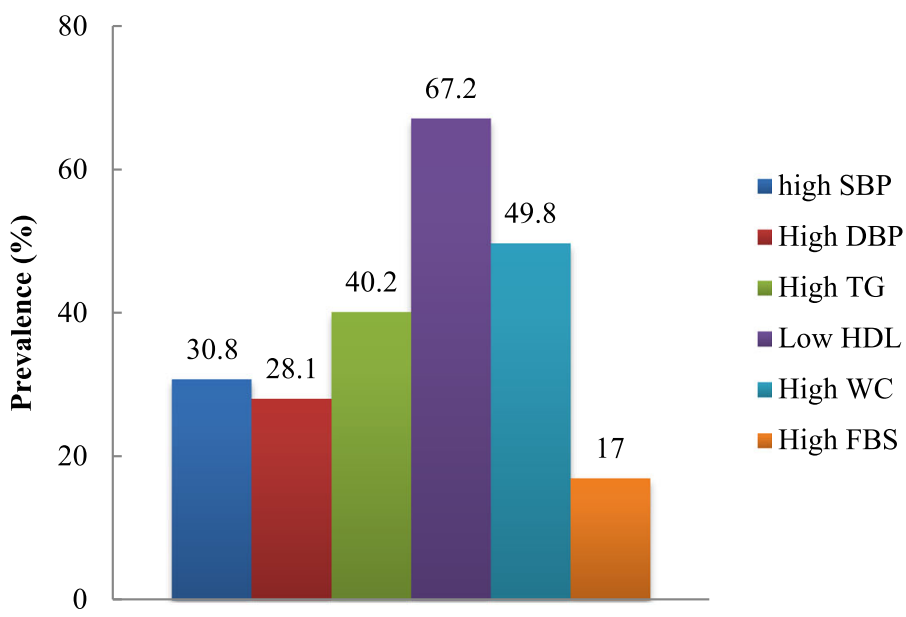

Fig. 1 Prevalence of components of metabolic syndrome across animal-sourced nutrient pattern (a), plant-sourced nutrient pattern (b) and mixed-source nutrient pattern (c). WC, waist circumference; TG, triglyceride; HDL-C, high-density lipoprotein cholesterol; FBS, fasting blood sugar; SBP, systolic blood pressure; DBP, diastolic blood pressure 
include a maximum number of MetS-associated nutrients and bioactive food compounds (40 in total).

In this cross-sectional study, we found a significant positive association between animal-sourced nutrient pattern (consisting of SFA, fat, protein, sugar, vitamins $\mathrm{B}_{5}, \mathrm{~B}_{12}, \mathrm{~B}_{2}$, A, K, biotin, phosphorus, cholesterol, selenium, sodium, PUFA, calcium and zinc) and odds of having MetS and high TG in study population. We also found a higher level of serum TG, AST, ferritin and SBP at the highest quartile of the animal-sourced nutrient pattern.

The relationship between isolated dietary consumptions of some nutrients in this nutrient pattern with MetS has been reported previously. Dietary intakes of SFA, sugar and cholesterol have been positively associated with MetS in previous studies [34, 35]. In contrast, some other nutrients in this pattern, such as vitamin C, selenium and zinc, have been inversely associated with MetS [36, 37]. In our study, SFA and PUFA, part of this nutrient pattern, were positively associated with risk of MetS but another study by Brady et al. [38] showed that there is no association between different dietary consumptions of omega-6 PUFAs and omega-3 PUFAs and the odds of MetS. Our results support the idea that a higher level of SFAs raises the risk of MetS.

Sugar and protein were also highly represented in animal-sourced nutrient pattern. In our study, protein was positively associated with MetS risk and high TG levels. In contrast to our results, several authors indicated that intake of protein-rich meals has some favorable effects such as regulation of appetite and increased thermogenesis which improves weight management and consequently decrease the risk of MetS and its components [39, 40]. A cohort study by Schulze et al. [41] demonstrated that higher intake of sugar-sweetened drinks may result in type 2 diabetes and MetS. Although there is some disagreement for some nutrients such as calcium and vitamin A, which is in agreement with our results, a cohort study conducted in France by Samara et al. [42] showed a positive and negative association for calcium levels with TG and HDL-c, respectively and Beydoun MA et al. [24] reported that vitamin A had a positive association with MetS and insulin resistance.

The existence of fat and sugar in this nutrient pattern may have resulted in a MetS-inducing effect; thus, it seems that sugar rise the odds of MetS even when coingested with nutrients that may contribute to protect against MetS. Therefore, the interaction of nutrients can affect their impact on MetS, and understanding these interactions in diets appears important.

In our study, plant-sourced nutrient pattern, which was positively correlated with total fiber, carbohydrate, vitamins $D, B_{6}, B_{3}, C, B_{1}, E$, magnesium, potassium, linoleic acid, and DHA, was negatively related to the prevalence of MetS, high SBP and high DBP. Also, those in the top quartile of the plant-sourced nutrient pattern had lower SBP, WC, WHR, LDL-C and FBS levels than participants in the lowest quartile. Dietary intakes of magnesium, fiber, vitamin $\mathrm{D}$ and potassium have been inversely associated with MetS and obesity in previous studies [43-45]. Some other nutrients in this pattern, such as vitamin $B_{3}$ [46] and $B_{1}$ [47] have been positively associated with obesity. B-vitamins may lead to increased appetite; therefore, their long-term intake may trigger unnecessary energy consumption and weight gain [48].

Possible mechanisms may be through improvement of glucose and insulin metabolism by vitamins $\left(E, B_{6}, B_{1}\right)$ and magnesium [49]; enhancement of endothelial function by vitamins $\left(\mathrm{B}_{3}\right.$ and $\left.\mathrm{E}\right)$ and magnesium [50]; reduced oxidative stress and increased HDL-C by magnesium and vitamins $\left(B_{3}\right.$ and $\left.E\right)$ [51] and reduction in blood pressure by magnesium and vitamins $\left(B_{3}, B_{1}\right)$ [50]. Magnesium acts as a cofactor for several enzymes and plays an important role in the insulin and glucose metabolism [49]. A recent meta-analysis of six cross-sectional studies presented that dietary magnesium consumption is inversely related to the prevalence of MetS [52].

Among dietary factors, fiber consumption could play an important role in the control of MetS. In line with our study, a cross-sectional study conducted in Japan showed an inverse relationship between MetS and potassium and dietary fiber [43]. It is well known that increased potassium consumption decreases blood pressure by neutralizing the effects of sodium [53]. A recent systematic review and meta-analysis reported that high dietary potassium consumption was related to lower odds of MetS [45] also another study showed a negative relationship between serum potassium level and the prevalence of MetS [26]. Contrary to our results, Iwasaki et al. [43] concluded that vitamin E positively related to the odds of MetS, and high blood pressure but Wei et al. [37] did not detect a significant relationship between the consumption of vitamins ( $\mathrm{E}$ and $\mathrm{A}$ ) and the development of MetS. It is possible that synergistic influence of fiber, magnesium, potassium and other nutrients in this pattern cause the significant effect of fiber on decreasing the risk of MetS.

Also, our finding revealed that the mixed-source nutrient pattern (high intake of fluoride, manganese, caffeine and folate) was positively related to MetS, high WC and SBP. Several prior animal studies have proposed that fluoride may affect glucose metabolism and insulin sensitivity $[54,55]$. Another cross-sectional study of 346 adults in northwestern Iran showed no relationship of fluoride in drinking water with WC, WHR and BMI [56]. The mechanisms by which fluoride interferes with MetS are unclear: nevertheless, fluoride may induce inflammation [57] and oxidative stress [58], all of which have been known to play an important role in obesity, hyperglycemia, dyslipidemia, and insulin resistance. Altogether, these risk factors significantly contribute to 
cardiovascular disease risk [59]. A study in Chinese adults over 18 years reported that higher manganese consumption was related to a reduced risk of the MetS in men $(\mathrm{OR}=0.62)$ but positively related to low HDL-C among both men and women [60]. Previous animal studies have shown that manganese consumption might decrease abdominal fat deposition by reducing glycerol in adipose tissue [61], malate dehydrogenase and fatty acid synthase activities in the liver [62], also decrease TC, HDL-C and LDL-C because manganese may be an important component of the lipoprotein structure [63].

Caffeine, part of nutrient pattern 3, was positively associated with MetS risk. In line with the results, Stutz et al. in a cross sectional study, reported that coffee consumption was related to higher risk of MetS in population of subjects with type 1 diabetes [64] but in a cross-sectional study by Wilsgaard et al. [65] coffee intake was not significantly associated with incidence of MetS. Contrary to our results, Hino et al. demonstrated that coffee intake has beneficial effects on SBP, DBP, FBS, TG levels, and WC [66]. Therefore, the interaction of nutrients can affect their impact on MetS, and understanding these interactions in diets appears important. In the current study, extracted nutrient patterns were different from those reported for African [67] and Chinese populations [68].

The discrepancy between the results of the studies may be due to the different characteristics of the study populations, included nutrients, and also the area where the study was carried out. Significant protective effect was detected with plant-sourced nutrient pattern high in total fiber, carbohydrate, vitamins $\mathrm{D}, \mathrm{B}_{6}, \mathrm{~B}_{3}, \mathrm{C}, \mathrm{B}_{1}, \mathrm{E}$, magnesium, potassium, linoleic acid, and DHA among nutrient patterns found in current study. Nutrient patterns via taking into account the mixture of several nutrients, their interaction and the synergistic effect can be a strong forecaster of risk MetS. The major strengths of our study are the relatively large sample size, population based design and use of validated questionnaires. The present study has some limitations. As this is a cross-sectional observation, no causal associations can be determined. Since nutrient consumption was evaluated using an FFQ, some amount of measurement error is unavoidable.

\section{Conclusion}

This present study confirms the important effect of nutrients on MetS risk. Our results suggest that adherence to the nutrient pattern rich in fiber, carbohydrate, vitamins $\mathrm{D}, \mathrm{B}_{6}, \mathrm{~B}_{3}$, $\mathrm{C}, \mathrm{B}_{1}, \mathrm{E}$, magnesium, potassium, linoleic acid, and DHA is associated with a lower risk of MetS, while animal- and mixed-sourced nutrient patterns are positively associated with greater odds of MetS; However, further longitudinal and interventional studies are required to make a clear conclusion.

\section{Abbreviations}

MetS: Metabolic syndrome; PA: Physical activity; PCA: Principal Components Analysis; BMI: Body mass index; CVD: Cardiovascular disease; WC: Aist circumference; TG: Triglyceride; TC: Total cholesterol; HDL-C: High-density lipoprotein cholesterol; FBS: Fasting blood sugar; SBP: Systolic blood pressure; DBP: Diastolic blood pressure; FFQ: Food frequency questionnaire: Cl: Confidence interval; IPAQ: International physical activity questionnaire; MET: Metabolic equivalent; SD: Standard Deviations; WHR: Waist hip ratio

\section{Acknowledgements}

We thank all of the study participants. This article was written based on the data set of the MSc thesis (Registration number: IR.TBZMED.REC.1398.077) registered at Tabriz University of Medical Sciences, Iran. The work is obtained from M.S. thesis of Mahdi Vajdi.

\section{Authors' contributions}

MA designed the study and served as a supervisor for this research. MV contributed to data collection and primary manuscript writing. LN assisted with data collection and also critically revised the manuscript. All authors read and approved the final version of the manuscript.

\section{Funding}

This document was part of an MSc thesis approved by Tabriz University of Medical Sciences, Iran (Registration number: IR.TBZMED.REC.1398.077). Current work has been financially supported by a grant from Tabriz University of Medical Sciences. The funders had no role in hypothesis generation, designing and recruiting the study. Their role was only financial supporting.

\section{Availability of data and materials}

All of the data are available with reasonable request from the corresponding author.

\section{Ethics approval and consent to participate}

Each participant was completely informed about the study protocol and provided a written informed consent form before taking part in the study. The study protocol was approved and registered by the ethics committee of Tabriz University of Medical Sciences (Ethics number: IR.TBZMED.REC. 1398.077)

\section{Consent for publication}

Not applicable.

\section{Competing interests}

The authors declare that there is no conflict of interest.

\section{Author details}

${ }^{1}$ Nutrition Research Center, Tabriz University of Medical Sciences, Tabriz, Iran. ${ }^{2}$ Drug Applied Research Center, Tabriz University of Medical Sciences, Tabriz, Iran. ${ }^{3}$ Tabriz Health Services Management Research Center, Health Management and Safety Promotion Research Institute, Tabriz University of Medical Sciences, Tabriz, Iran.

Received: 12 January 2020 Accepted: 6 May 2020

Published online: 19 May 2020

References

1. Eckel RH, Grundy SM, Zimmet PZ. The metabolic syndrome. Lancet. 2005; 365(9468):1415-28.

2. Ranasinghe $P$, Mathangasinghe $Y$, Jayawardena R, Hills A, Misra A Prevalence and trends of metabolic syndrome among adults in the asiapacific region: a systematic review. BMC Public Health. 2017;17(1):101.

3. Dalvand S, Niksima SH, Meshkani R, Gheshlagh RG, Sadegh-Nejadi S, Kooti W, Parizad N, Zahednezhad H, Afrisham R. Prevalence of metabolic syndrome among Iranian population: a systematic review and meta-analysis. Iran J Public Health. 2017;46(4):456.

4. Srikanthan K, Feyh A, Visweshwar H, Shapiro Jl, Sodhi K. Systematic review of metabolic syndrome biomarkers: a panel for early detection, management, and risk stratification in the west Virginian population. Int J Med Sci. 2016;13(1):25-38. 
5. Lutsey PL, Steffen LM, Stevens J. Dietary intake and the development of the metabolic syndrome. Circulation. 2008;117(6):754-61.

6. Nava LT, Zambrano JM, Arviso KP, Brochetti D, Becker KL. Nutrition-based interventions to address metabolic syndrome in the $\mathrm{N}$ avajo: a systematic review. J Clin Nurs. 2015;24(21-22):3024-45.

7. Guo H, Niu K, Monma H, Kobayashi Y, Guan L, Sato M, Minamishima D, Nagatomi R. Association of Japanese dietary pattern with serum adiponectin concentration in Japanese adult men. Nutr Metab Cardiovasc Dis. 2012;22(3):277-84.

8. Hong $S$, Song $Y$, Lee $K H$, Lee $H S$, Lee $M$, Jee $S H$, Joung $H$. A fruit and dairy dietary pattern is associated with a reduced risk of metabolic syndrome. Metabolism. 2012;61 (6):883-90.

9. Castanho GKF, Marsola FC, Mclellan KCP, Nicola M, Moreto F, Burini RC. Consumption of fruit and vegetables associated with the metabolic syndrome and its components in an adult population sample. Cien Saude Colet. 2013;18(2):385-92.

10. Huang $\mathrm{H}-\mathrm{Y}$, Korivi M, Tsai C-H, Yang J-H, Tsai Y-C. Supplementation of lactobacillus plantarum K68 and fruit-vegetable ferment along with high fat-fructose diet attenuates metabolic syndrome in rats with insulin resistance. Evid Based Complement Alternat Med. 2013;2013:943020.

11. Fulgoni VL, Dreher M, Davenport AJ. Avocado consumption is associated with better diet quality and nutrient intake, and lower metabolic syndrome risk in US adults: results from the National Health and nutrition examination survey (NHANES) 2001-2008. Nutr J. 2013;12(1):1.

12. Vega GL. Obesity, the metabolic syndrome, and cardiovascular disease. Am Heart J. 2001;142(6):1108-16.

13. Marshall J, Bessesen D, Hamman R. High saturated fat and low starch and fibre are associated with hyperinsulinaemia in a non-diabetic population: the San Luis Valley diabetes study. Diabetologia. 1997;40(4):430-8.

14. Hu FB. Dietary pattern analysis: a new direction in nutritional epidemiology. Curr Opin Lipidol. 2002;13(1):3-9.

15. Newby P, Tucker KL. Empirically derived eating patterns using factor or cluster analysis: a review. Nutr Rev. 2004;62(5):177-203.

16. Sahyoun NR, Jacques PF, Zhang XL, Juan W, Mckeown NM. Whole-grain intake is inversely associated with the metabolic syndrome and mortality in older adults. Am J Clin Nutr. 2006;83(1):124-31.

17. Esmaillzadeh A, Kimiagar M, Mehrabi Y, Azadbakht L, Hu FB, Willett WC. Fruit and vegetable intakes, $\mathrm{C}$-reactive protein, and the metabolic syndrome. Am J Clin Nutr. 2006;84(6):1489-97.

18. Kastorini C-M, Milionis HJ, Esposito K, Giugliano D, Goudevenos JA, Panagiotakos DB. The effect of Mediterranean diet on metabolic syndrome and its components: a meta-analysis of 50 studies and 534,906 individuals. J Am Coll Cardiol. 2011;57(11):1299-313.

19. Asghari G, Yuzbashian E, Mirmiran P, Hooshmand F, Najafi R, Azizi F. Dietary approaches to stop hypertension (DASH) dietary pattern is associated with reduced incidence of metabolic syndrome in children and adolescents. J Pediatr. 2016;174:178-84 e1.

20. Rodríguez-Monforte M, Sánchez E, Barrio F, Costa B, Flores-Mateo G. Metabolic syndrome and dietary patterns: a systematic review and metaanalysis of observational studies. Eur J Nutr. 2017;56(3):925-47.

21. Alles B, Samieri C, Lorrain S, Jutand M-A, Carmichael P-H, Shatenstein B, Gaudreau P, Payette H, Laurin D, Barberger-Gateau P. Nutrient patterns and their food sources in older persons from France and Quebec: dietary and lifestyle characteristics. Nutrients. 2016;8(4):225.

22. Liu S, Song Y, Ford ES, Manson JE, Buring JE, Ridker PM. Dietary calcium, vitamin $\mathrm{D}$, and the prevalence of metabolic syndrome in middle-aged and older US women. Diabetes Care. 2005;28(12):2926-32.

23. Saltevo J, Niskanen L, Kautiainen H, Teittinen J, Oksa H, Korpi-Hyövälti E, Sundvall J, Männistö S, Peltonen M, Mäntyselkä P, et al. Serum calcium level is associated with metabolic syndrome in the general population: FIN-D2D study. Eur J Endocrinol. 2011;165(3):429-34.

24. Beydoun MA, Canas JA, Beydoun HA, Chen X, Shroff MR, Zonderman $A B$. Serum antioxidant concentrations and metabolic syndrome are associated among US adolescents in recent national surveys. J Nutr. 2012;142(9):1693-704

25. Sluiis I, Beulens JW, Grobbee DE, van der Schouw YT. Dietary carotenoid intake is associated with lower prevalence of metabolic syndrome in middle-aged and elderly men. J Nutr. 2009;139(5):987-92.

26. Sun $K$, Su T, Li M, Xu B, Xu M, Lu J, et al. Serum potassium level is associated with metabolic syndrome: a population-based study. Clin Nutr. 2014:33(3):521-7.
27. Khayyatzadeh SS, Moohebati M, Mazidi M, Avan A, Tayefi M, Parizadeh SMR, Ebrahimi M, Heidari-Bakavoli A, Azarpazhooh MR, Esmaily H, et al. Nutrient patterns and their relationship to metabolic syndrome in Iranian adults. Eur J Clin Investig. 2016:46(10):840-52.

28. Tabrizi JS, Farahbakhsh M, Sadeghi-Bazargani H, Nikniaz L. Prevention and control of non-communicable diseases in Iranian population: life style promotion project phase II: study protocol. Iran J Public Health. 2018;47(9): 1397.

29. Vasheghani-Farahani A, Tahmasbi M, Asheri H, Ashraf H, Nedjat S, Kordi R. The Persian, last 7-day, long form of the international physical activity questionnaire: translation and validation study. Asian J Sports Med. 2011; 2(2):106.

30. Nikniaz L, Tabrizi J, Sadeghi-Bazargani H, Farahbakhsh M, Tahmasebi S, Noroozi S. Reliability and relative validity of short-food frequency questionnaire. Br Food J. 2017;119(6):1337-48.

31. Friedewald WT, Levy RI, Fredrickson DS. Estimation of the concentration of low-density lipoprotein cholesterol in plasma, without use of the preparative ultracentrifuge. Clin Chem. 1972;18(6):499-502.

32. Huang X-J, Choi Y-K, Im H-S, Yarimaga O, Yoon E, Kim H-S. Aspartate aminotransferase (AST/GOT) and alanine aminotransferase (ALT/GPT) detection techniques. Sensors (Basel). 2006;6(7):756-82.

33. Grundy SM, Cleeman JI, Daniels SR, Donato KA, Eckel RH, Franklin BA, Gordon DJ, Krauss RM, Savage PJ, Smith SC Jr, et al. Diagnosis and management of the metabolic syndrome: an American Heart Association/ National Heart, Lung, and Blood Institute scientific statement. Circulation. 2005;112(17):2735-52.

34. Hosseinpour-Niazi S, Mirmiran P, Fallah-Ghohroudi A, Azizi F. Combined effect of unsaturated fatty acids and saturated fatty acids on the metabolic syndrome: Tehran lipid and glucose study. J Health Popul Nutr. 2015;33(1):5.

35. Seo EH, Kim H, Kwon O. Association between Total sugar intake and metabolic syndrome in middle-aged Korean men and women. Nutrients. 2019;11(9):2042.

36. Li Y, Guo H, Wu M, Liu M. Serum and dietary antioxidant status is associated with lower prevalence of the metabolic syndrome in a study in Shanghai, China. Asia Pac J Clin Nutr. 2013;22(1):60.

37. Wei J, Zeng C, Gong Q-Y, Li X-X, Lei G-H, Yang T-B. Associations between dietary antioxidant intake and metabolic syndrome. PLoS One. 2015;10(6): e0130876.

38. Brady LM, Williams CM, Lovegrove JA. Dietary PUFA and the metabolic syndrome in Indian Asians living in the UK. Proc Nutr Soc. 2004;63(1):115-25.

39. Leidy HJ, Clifton PM, Astrup A, Wycherley TP, Westerterp-Plantenga MS, Luscombe-Marsh ND, Woods SC, Mattes RD. The role of protein in weight loss and maintenance. Am J Clin Nutr. 2015;101(6):1320S-9S.

40. Keller U. Dietary proteins in obesity and in diabetes. Int J Vitam Nutr Res. 2011;81(2):125.

41. Schulze MB, Manson JE, Ludwig DS, Colditz GA, Stampfer MJ, Willett WC, Hu FB. Sugar-sweetened beverages, weight gain, and incidence of type 2 diabetes in young and middle-aged women. JAMA. 2004;292(8): 927-34

42. Samara A, Herbeth B, Ndiaye NC, Fumeron F, Billod S, Siest G, Visvikis-Siest S. Dairy product consumption, calcium intakes, and metabolic syndromerelated factors over 5 years in the STANISLAS study. Nutrition. 2013;29(3): $519-24$.

43. Iwasaki Y, Arisawa K, Katsuura-Kamano S, Uemura H, Tsukamoto M, Kadomatsu Y, Okada R, Hishida A, Tanaka K, Hara M, et al. Associations of nutrient patterns with the prevalence of metabolic syndrome: results from the baseline data of the Japan multi-institutional collaborative cohort study. Nutrients. 2019:11(5):990

44. Ford ES, Ajani UA, McGuire LC, Liu S. Concentrations of serum vitamin D and the metabolic syndrome among US adults. Diabetes Care. 2005;28(5): $1228-30$.

45. Cai X, Li X, Fan W, Yu W, Wang S, Li Z, Scott EM, Li X. Potassium and obesity/metabolic syndrome: a systematic review and meta-analysis of the epidemiological evidence. Nutrients. 2016;8(4):183.

46. Da Li W-PS, Zhou Y-M, Liu Q-G, Zhou S-S, Luo N, Bian F-N, Zhao ZG, Guo M. Chronic niacin overload may be involved in the increased prevalence of obesity in US children. World J Gastroenterol. 2010;16(19):2378.

47. Zhou S-S, Li D, Zhou Y-M, Sun W-P, Liu Q-G. B-vitamin consumption and the prevalence of diabetes and obesity among the US adults: population based ecological study. BMC Public Health. 2010;10(1):746. 
48. Institute of Medicine (US) Standing Committee on the Scientific Evaluation of Dietary Reference Intakes. Dietary Reference Intakes for Thiamin, Riboflavin, Niacin, Vitamin B6, Folate, Vitamin B12, Pantothenic Acid, Biotin,and Choline. Cambridge: National Academies Press (US); 1998.

49. Saris N-EL, Mervaala E, Karppanen H, Khawaja JA, Lewenstam A. Magnesium: an update on physiological, clinical and analytical aspects. Clin Chim Acta. 2000;294(1-2):1-26.

50. Ahmadi A, Gharipour M, Arabzadeh G, Moin P, Hashemipour M, Kelishadi R. The effects of vitamin $E$ and omega-3 PUFAs on endothelial function among adolescents with metabolic syndrome. Biomed Res Int. 2014;2014:906019.

51. Costacou T, Levy AP, Miller RG, Snell-Bergeon J, Asleh R, Farbstein D, Fickley CE, Pambianco G, de la Vega R, Evans RW, Orchard TJ. Effect of vitamin E supplementation on $\mathrm{HDL}$ function by haptoglobin genotype in type 1 diabetes: results from the HapE randomized crossover pilot trial. Acta Diabetol. 2016:53(2):243-50.

52. Dibaba DT, Xun P, Fly AD, Yokota K, He K. Dietary magnesium intake and risk of metabolic syndrome: a meta-analysis. Diabet Med. 2014;31 (11):1301-9.

53. Filippini T, Violi F, D'Amico R, Vinceti M. The effect of potassium supplementation on blood pressure in hypertensive subjects: a systematic review and meta-analysis. Int J Cardiol. 2017;230:127-35.

54. Pereira AG, Chiba FY, MSdLC M, Pereira RF, RdCA N, TVS T, Okamoto R, Sumida DH. Effects of fluoride on insulin signaling and bone metabolism in ovariectomized rats. J Trace Elem Med Biol. 2017;39:140-6.

55. Hu C-y, L-q R, Li X-n WN, G-s L, Liu Q-y XH. Effect of fluoride on insulin leve of rats and insulin receptor expression in the MC3T3-E1 cells. Biol Trace Elem Res. 2012:150(1-3):297-305.

56. Yousefi M, Yaseri M, Nabizadeh R, Hooshmand E, Jalilzadeh M, Mahvi AH, Mohammadi AA. Association of hypertension, body mass index, and waist circumference with fluoride intake; water drinking in residents of fluoride endemic areas, Iran. Biol Trace Elem Res. 2018;185(2):282-8.

57. Afolabi OK, Oyewo EB, Adekunle AS, Adedosu OT, Adedeji AL. Oxidative indices correlate with dyslipidemia and pro-inflammatory cytokine levels in fluoride-exposed rats. Arh Hig Rada Toksikol. 2013;64(4):521-9.

58. Lu Y, Luo Q, Cui H, Deng H, Kuang P, Liu H, Fang J, Zuo Z, Deng J, Li Y, Wang $X$. Sodium fluoride causes oxidative stress and apoptosis in the mouse liver. Aging (Albany NY). 2017;9(6):1623.

59. Siti HN, Kamisah Y, Kamsiah J. The role of oxidative stress, antioxidants and vascular inflammation in cardiovascular disease (a review). Vasc Pharmacol. 2015;71:40-56.

60. Zhou B, Su X, Su D, Zeng F, Wang MH, Huang L, Huang E, Zhu Y, Zhao D, He $D$, Zhu X. Dietary intake of manganese and the risk of the metabolic syndrome in a Chinese population. Br J Nutr. 2016;1 16(5):853-63.

61. Baquer NZ, Sinclair M, Kunjara S, Yadav UC, McLean P. Regulation of glucose utilization and lipogenesis in adipose tissue of diabetic and fat fed animals: effects of insulin and manganese. J Biosci. 2003;28(2):215-21.

62. Wang $M$, Chen Z, Zhang L, Luo X. Effect of different manganese sources on activities and gene expression of key enzymes in fat metabolism of broilers. Sci Agri Sin. 2011:44(18):3850-8

63. Lee Y-K, Lyu E-S, Oh S-Y, Park H-R, Ro H-K, Heo Y-R, Hyun T, Choi MK. Daily copper and manganese intakes and their relation to blood pressure in normotensive adults. Clin Nutr Res. 2015;4(4):259-66.

64. Stutz B, Ahola A, Harjutsalo V, Forsblom C, Groop P-H, Group FS. Association between habitual coffee consumption and metabolic syndrome in type 1 diabetes. Nutr Metab Cardiovasc Dis. 2018;28(5):470-6.

65. Wilsgaard T, Jacobsen BK. Lifestyle factors and incident metabolic syndrome: the Tromsø study 1979-2001. Diabetes Res Clin Pract. 2007;78(2):217-24.

66. Hino A, Adachi H, Enomoto M, Furuki K, Shigetoh Y, Ohtsuka M, Kumagae SI, Hirai Y, Jalaldin A, Satoh A, et al. Habitual coffee but not green tea consumption is inversely associated with metabolic syndrome: an epidemiological study in a general Japanese population. Diabetes Res Clin Pract. 2007;76(3):383-9.

67. Chikowore T, Pisa P, van Zyl T, Feskens E, Wentzel-Viljoen E, Conradie K. Nutrient patterns associated with fasting glucose and glycated haemoglobin levels in a black south African population. Nutrients. 2017;9(1):9.

68. Bian S, Gao Y, Zhang M, Wang X, Liu W, Zhang D, et al. Dietary nutrient intake and metabolic syndrome risk in Chinese adults: a case-control study. Nutr J. 2013;12(1):106.

\section{Publisher's Note}

Springer Nature remains neutral with regard to jurisdictional claims in published maps and institutional affiliations.

\section{Ready to submit your research? Choose BMC and benefit from}

- fast, convenient online submission

- thorough peer review by experienced researchers in your field

- rapid publication on acceptance

- support for research data, including large and complex data types

- gold Open Access which fosters wider collaboration and increased citations

- maximum visibility for your research: over $100 \mathrm{M}$ website views per year

At BMC, research is always in progress.

Learn more biomedcentral.com/submissions 